\title{
The Multigenerational Legacy of Diabetes, Emotional Reactions, and Insulin Adherence
}

\author{
Melissa Scollan-Koliopoulos ${ }^{1}$ \\ ${ }^{1}$ Division of Primary Care Mental Health, Transformative Health and \\ Behavior Solutions, Ringwood, New Jersey, United States
}

Address for correspondence Melissa Scollan-Koliopoulos, EdD, APN-C, CDE, Division of Primary Care Mental Health, Transformative Health and Behavior Solutions, Ringwood 07456, New Jersey, United States (e-mail: ms2039@columbia.edu).

\begin{abstract}
Keywords

- diabetes

- insulin

- self-care

- coping

- illness perceptions

- multigenerational legacy of diabetes

Purpose The purpose of this study was to estimate the effect of having a multigenerational legacy of diabetes on insulin adherence by measuring memories of family members' experiences with insulin self-injection.

Methods A cross-sectional correlational design was implemented by surveying hospitalized adults with type 2 diabetes who recently transitioned to using insulin. Surveys included items about the vividness, frequency, and controllability of memories of a family member self-injecting insulin and concordant emotional reaction, and one's own emotional reaction to a diagnosis of diabetes and insulin regimen adherence.

Results A diverse sample responded to the surveys. Significant associations were revealed between having a recollection of a family member fearing insulin injections and a negative emotional reaction toward one's own diabetes diagnosis with suboptimal insulin adherence. Suboptimal insulin adherence may be an indication of avoidance-based coping. Inquiry into how a multigenerational legacy of diabetes influences individual coping styles for diabetes self-management will help clinicians identify patients at risk for suboptimal insulin adherence.
\end{abstract}

\section{Introduction}

Type 2 diabetes is reaching epidemic proportions and is associated with a hereditary predisposition. ${ }^{1}$ Individuals with a family history of diabetes who subsequently develop diabetes themselves typically have an awareness of family members' experiences with diabetes. The awareness of how a family member coped with self-management regimens has the potential to influence one's own self-care behavior. This phenomenon is known as a multigenerational legacy of diabetes. ${ }^{2}$ One area of particular concern is insulin adherence in the context of a multigenerational legacy of diabetes, whereby recollections of a family member's experience with insulin contribute to one's own suboptimal adherence. ${ }^{3}$ The insulin syringe is often an object of anxiety in diabetes care.
The disease trajectory for the most common form of diabetes, a noninsulin dependent, yet sometimes requiring or benefitting type 2 diabetes is often viewed as an end point or last resort. Typically, patients exert an effort avoid insulin for various reasons. The effort is often laden with emotional sequalae, termed psychological insulin resistance in the DAWN study. ${ }^{4}$ This is a report on a study that tested the effect of recollections of family members' emotional reaction to self-injecting insulin and its effect on coping with diabetes and insulin regimen adherence.

Adherence to insulin is poor with few patient-perceived barriers, other than inflexible regimens significantly explaining the variance. ${ }^{4}$ Medication nonadherence is a major public health concern in the United States, with suboptimal

(c) 2020. Novo Nordisk Education Foundation.

This is an open access article published by Thieme under the terms of the Creative Commons Attribution-NonDerivative-NonCommercial-License, permitting copying and reproduction so long as the original work is given appropriate credit. Contents may not be used for commercial purposes, or adapted, remixed, transformed or built upon. (https://creativecommons.org/licenses/by-nc-nd/4.0/).

Thieme Medical and Scientific Publishers Pvt. Ltd. A-12, 2nd Floor, Sector 2, Noida-201301 UP, India 
adherence to diabetes medication being associated with hospitalization, taking second place to that of heart failure. ${ }^{5}$ With diabetes medication adherence ranging from 13 to $64 \%,{ }^{6}$ there is reason for concern that public health efforts to control the rise of diabetes-related complications may be undermined. The United Kingdom Prospective Diabetes Study demonstrated that for each $1 \%$ reduction in average glycosylated hemoglobin, there is a $21 \%$ reduction in the risk of any diabetes-related complication. ${ }^{7}$ Given the epidemic and progression of diabetes in the United States, it is imperative that clinicians address medication adherence as the means to complication prevention as a matter of routine public health significance. $^{8}$

Fear of injection, ${ }^{4}$ embarrassment, ${ }^{4,9}$ and emotional problems $^{9}$ are consistently cited as possible explanations for insulin omission. Few studies articulated the antecedent factors leading to fear of injections or have addressed the consequences of fear by observing family members who required medication by injection 1 day. One contributing factor to regimen nonadherence that can be addressed includes having a multigenerational legacy of diabetes. According to the theoretical foundation of multigenerational legacies of diabetes, a family member's self-care behavior may be vividly recalled. ${ }^{3}$ Individuals witness a family member's self-injection of insulin and observe concordant emotional reactions. The observation of the coping style of a family member leaves an impression of coping options (i.e., acceptance or avoidance) and the potential to emulate self-care behavior. ${ }^{3}$ Furthermore, insulin use is associated with drug adverse events (i.e., hypoglycemic-induced confusion, seizures, or loss of consciousness) that may result in hospitalization and/or mortality. Family members often observe the consequences of adverse drug events, experience emotional reactions to the observation, and accommodate their own coping pattern that will ultimately influence one's own diabetes self-management.

Memory activation is posited to result in vivid recollections of attributes (people, place, activity, and source) based on events deemed important (personally consequential) and encoded within emotional reactions/affect (surprise, intensity, valence) with rehearsal (repeated thoughts of the event). ${ }^{10}$ For example, the approach/avoidance influence on behavioral outcomes in the context of a multigenerational legacy of diabetes has been observed with foot self-care engagement. ${ }^{11}$ Individuals may be prone to decisional conflict and trepidation resulting in an approach/avoidance pattern of behavior whereby insulin use may be sporadic depending upon memory activation and subsequent emotional reactions to the memory.

The central hypothesis of this study is that the patient experiencing fear similar to the family member's fear makes insulin regimen acceptance unlikely (i.e., feedback-based learning theory ${ }^{12,13}$ ) or accepted with some degree of trepidation that will ultimately contribute to a likelihood of insulin omission. Based on Rolland's (1994) concept of a multigenerational legacy of illness, individuals anticipate a similar disease trajectory to that of a family member. ${ }^{14}$
Based on the preceding literature review and theoretical foundation, the following hypotheses were tested:

Hypothesis 1: Individuals who remember a family member having an emotional reaction toward insulin use will have vivid, frequent, and uncontrollable memories of that family member self-injecting insulin.

Hypothesis 2: Individuals who have vivid, frequent, and uncontrollable memory rehearsal of the family member self-injecting insulin will experience emotional reactions (arousal) that may result in suboptimal insulin adherence (avoidance).

Hypothesis 3: Individuals who experience vivid, frequent, and uncontrollable memories of a family member self-injecting insulin will experience approach/ avoidance-based coping over his/her own insulin regimen adherence.

\section{Research Design and Methodology}

This is a report of an observational correlational design with purposive sampling to estimate the effect of memory rehearsal and emotional reactions relevant to a multigenerational legacy of diabetes on insulin adherence. The correlational design is useful for estimating the degree of variance accounted for by predictor variables on outcomes measures.

\section{Subject Recruitment}

Approximately 250 individuals were invited to participate yielding an $80 \%$ participation rate. The participation rate was high presumably because nurses engaged the patients prior to the researcher informing them of the study. Participants $(n=200)$ were adults recruited from three Northeast coast hospitals in the United States. All participants had type 2 diabetes, a family history of diabetes, and were new to or recently transitioned to the use of insulin. Excluded from participation were those under 18 years of age (due to parental role in medication adherence); affected by critical illness, acute pain, type 1 diabetes (because they are dependent of insulin), and/or pregnancy; and those unable to provide informed consent. Those hospitalized over 8 days or those who reported being too ill to adhere to their usual diabetes regimen the week before hospitalization were also excluded from participation. Participants received a $\$ 40$ gift card as a token of appreciation for completing the surveys. The study was approved by the Rutgers-UMDNJ-Legacy Institutional Review Board and the human subject protection committee at each of the recruitment sites.

\section{Measures}

Paper-and-pencil surveys were administered to those who self-reported having insulin-requiring diabetes. All data was collected during hospitalization and participants were given approximately 1 hour to respond, accounting for routine hospital interruptions. To ensure clarity, participants were instructed to "only think about the family member's experiences" on a certain color form and their "own experiences" on a different colored form. The questions were framed in a 
way to allow respondents to differentiate perceptions of self as opposed to those of a family member.

\section{Recollections of Family Diabetes Experiences Survey}

The survey includes items that measure the degree to which an individual remembers a specific family member's engagement in diabetes self-care. The survey measures the vividness, frequency, and ability to control one's ability to stop the memory from occurring. The items are individually composite scored for each memory (the distinct memories [i.e., diet and pill taking] are not intended to be combined as a subscale). Respondents are asked to remember only one blood relative they remember the most (parent, grandparent, sibling, aunt/uncle, or cousin). The items included (1) I can remember my relative: "Taking an insulin shot" (no recall, very little recall, some recall, strong recall) with a high score indicating vividness; (2) On how many of the past 7 days did you recall your relative taking an insulin shot (0-7) with a higher number indicating more weekly frequency; and (3) "How certain are you that you can make yourself stop thinking of your family member taking an insulin shot (very unsure, unsure, neither sure or unsure, sure, very sure)" with high scores indicating an ability to control the memories. A factor analysis to estimate the reliability of the three-item subscale revealed factor loadings over 0.80 for each item and a Cronbach's $\alpha$ of 0.90 for the composite. Other items included a specific subscale intended to measure a recollection of a family member's fear of insulin. Respondents were asked to rate how strongly they remember: "My family member with diabetes was: Afraid to go on insulin; was fearful of needing to give self-injections (shots) and; was afraid insulin would cause complications." A factor analysis using orthogonal rotation on this sample revealed that with maintaining items with a loading of over 0.80 (consistent with Steven's reliability suggestions for small samples ${ }^{15}$ ), a Cronbach's $\alpha$ of 0.82 was estimated.

\section{Summary of Diabetes Self-Care Activities Survey}

The most widely utilized and validated diabetes self-care measure by Toobert et al measures regimen adherence by controlling for subjectivity and impression management by asking individuals how many of the past 7 days that he/ she felt well, did they fully comply with their prescriber's orders. ${ }^{16}$ In this survey, only the single-item insulin injection was used to measure the study participant's adherence with insulin as a proxy of approach/avoidance-based coping. Participants were asked to think about the 7 days they felt well before being admitted.

\section{Brief Illness Perception Questionnaire}

The emotional consequences subscale of the Brief Illness Perception Questionnaire by Broadbent was used to estimate negative and positive mood scores as a reaction to having diabetes. The single item has been validated in previous studies on a 1 to 10 scale with high scores indicating more emotional consequences of diabetes. The item used read, "How much does your illness affect you emotionally? (e.g., Does it make you angry, scared, upset, or depressed?)." The illness perception constructs have been shown to be predictive of diabetes self-care behavior in previous studies on those with a family history of diabetes..$^{11,17,18}$

\section{Data Analysis}

Data analyses were completed using SPSS version 18.0. ${ }^{19}$ Descriptive data was estimated using mean and standard deviation calculations. Multiple hierarchical linear regression analysis was used because it is helpful in explaining the percentage of variance of a predictor variable associated with an outcome variable while controlling for co-variation. Data was evaluated for normality and violations of regression analysis. However, to reduce the chances of a type 1 error and inflated correlation coefficients, we took precautionary measures when conducting our statistical analyses (i.e., modified Sheffé method, initial analyses accept an $\alpha$ of 0.05 , followed by 0.01 and $0.001^{20}$ ). The measures were validated using orthogonal factor rotation and internal consistency reliability was estimated for each subscale using strict criteria for small samples (i.e., accepted loadings included those over 0.80).

\section{Results}

\section{Participants}

Data sets returned from 92 subjects with type 2 diabetes and a family history of diabetes were completed and used in the analysis. All the subjects were newly initiated onto insulin within the past 6 months of hospitalization. The demographics included $43 \%$ White, $44 \%$ Black, 20\% Hispanic, and 15\% mixed, unknown, and "other" race/ethnicity, with $51 \%$ of the sample being male. Approximately $31 \%$ of the sample was young, in the range of 26 to 49 years of age. The sample was diverse in socioeconomic status with $36 \%$ earning incomes below $\$ 20,000$ a year and $30 \%$ reporting not completing high school.

\section{Vivid, Frequent, and Uncontrollable Memory Rehearsal and Negative Emotions}

Hypothesis 1: Individuals who remember a family member having an emotional reaction toward insulin use will have vivid, frequent, and uncontrollable memories of that family member self-injecting insulin. A positive association between the memory of a family member who "feared" insulin injections and those who had strong, frequent, and/or uncontrollable memories of those family members taking insulin shots $\left(R^{2}=.26, F[1.48]=16.23, p \leq 0.0001\right)$ was detected. Results of the regression analysis indicate that those who remember a family member being afraid to use insulin and who also have their own high scores on negative emotional reactions to having diabetes are likely to rehearse the memory of the family member self-injecting insulin $(F[2,136]=8.8$, $p \leq 0.000$ ). In this analysis, memories of the family member being fearful of insulin use contributed unique variance ( $\beta=0.31, p=0.000$ ) over and above one's own emotional reactions in the rehearsal of the vivid, frequent, and uncontrollable memories. 
In other words, the emotional reactions of the family member left an impression of the individual that will influence memory rehearsal irrelevant of one's own emotional state. In actuality, one may empathize with or acknowledge another's feelings, yet not endorse the same feelings.

\section{Vivid, Frequent, and Uncontrollable Memories,} Negative Emotions, and Insulin Adherence

Hypothesis 2: Individuals who have vivid, frequent, and uncontrollable memories of a family member self-injecting insulin will experience emotional reactions (arousal) that may result in suboptimal insulin adherence (avoidance). Having memories of a family member being fearful of using insulin has a direct effect on insulin adherence $(F[1,156]=5.5, \beta=0.18, p=0.02)$. This reveals that having memories of a family member being fearful of using insulin has a direct effect on insulin adherence, such that emotional reactions result in suboptimal insulin adherence. Alternatively, those who have little recollection of a family member having emotional reactions toward insulin use had less insulin omission and more regimen adherence.

Hypothesis 3: Individuals who experience vivid, frequent, and intrusive memories of a family member self-injecting insulin will result in the individual experiencing approach/avoidance-based coping with his/her own insulin regimen. Using a hierarchical approach, the next analysis involved the addition of scores on the subjects' own emotional reaction to having diabetes to the regression model. Results continued to show a significant relationship with the subjects' insulin adherence $(F[2,157]=3.3, p=0.04)$ with the memories of the family member being fearful of insulin injection continuing to contribute unique variance ( $\beta=0.19, p=0.02$ ). One's own emotional reactions to having diabetes did not contribute uniquely to the model $(\beta=0.08)$. In a third step, rehearsal scores were added to the model, collectively explained 95\% of the variance in insulin adherence $(F[3,125]=$ $388, p \leq 0.000)$ in this sample with rehearsal scores contributing uniquely $(\beta=0.95, p=0.000)$ along with memories of the family member being fearful of insulin ( $\beta=0.06, p=0.03$ ), and the individuals own emotional reaction to having diabetes ( $\beta=-0.06, p=0.02$ ). This indicates that those lacking an emotional reaction to having diabetes, those unlikely to have had a family member fearful of insulin injection, and those who rehearse the memories of the family member self-injecting insulin are likely to approach their insulin regimen with adherence. Likewise, those who experience negative emotional reactions, have memories of a family member being fearful of insulin injection, and rehearse memories of the family member self-injecting insulin avoid insulin regimen adherence. Both subsets of the sample rehearsed the memories or had vivid, frequent, and uncontrollable memories of a family member injecting insulin. However, those who endorsed a family member with a negative emotional reaction preceding one's own negative emotional reaction were influenced by memory rehearsal in such a way that he/she avoided insulin. On the converse, those who did not recall negative emotions of a family member and whose own experience did not include negative emotions were more likely to demonstrate insulin regimen adherence. It's as if the awareness of emotions can be handed down and shape one's own self-care outcomes.

\section{Discussion}

This study revealed that emotional reactions to diabetes are associated with how individuals cope with memories (i.e., rehearsal) of a family member's experiences with a hereditary illness and its treatment (approach/avoidance with regimen adherence). Ruminations are repetitive thoughts for which there is typically no attempt by the individual to stop them. Individuals tend to assume that their memories are exact replicas of events or realities that once existed in time, rather than realizing that subjective elements, such as judgment, inference, emotion, and perception, play large roles in the construction and reconstruction of memories. ${ }^{21-23}$ In the case of a multigenerational legacy of diabetes, the visual image of a family member injecting insulin may have been formed with an awareness of the family member having a negative attitude toward using insulin, making the situation stressful for the observer. Some recollections of a family member's experiences with diabetes may be vague (difficult to remember in detail; lacking clarity; involving effort to retrieve memory), whereas others may be quite vivid (easily recalled details; very clear mental picture; little effort to retrieve memory). For some individuals, unpleasant events may be easily recalled because they are vivid or have had a traumatic effect on the witness resulting in repetitive arousal (i.e., fear). ${ }^{24,25}$ For example, remembering a family member become unconscious after taking an insulin shot may be traumatic because it poses an actual threat of unnatural death. Another example may be simply that the individual was empathetic to the family member's fear and/or negative attitudes and internalized feelings as the memory of that family member injecting insulin was being stored. Some familial memories go beyond being merely vivid and become intrusive (unwanted or recalled at inopportune times; may or may not cause distress for an individual). Intrusive memories are those memories that are undesirable and can be retrieved at times with little effort. ${ }^{25}$

Some individuals are more vulnerable to rehearsal of events (rumination) to try to make sense of the situation and this can result in reiteration of a memory and has been associated with recurrent, spontaneous, and often uncontrollable thoughts (i.e., intrusion) in certain individuals. ${ }^{25,26}$

Memory vividness is influenced by the emotional context (i.e., fear) within which the memory is formed with consequential events. ${ }^{27}$ The emotional context may be a direct 
result of the family member's outward display of emotions that becomes internalized by the individual patient (i.e., needle fear) or may result from the patient's own emotional reaction toward witnessing the family member's insulin self-injection (i.e., disgust). In other words, memories that are formed under stressful conditions are likely to be easily recalled. It is also possible that an individual take on the family member's negative attitudes toward insulin (i.e., a suggestion). Events recalled with ease due to their vividness and content-rich detail have the potential to be rehearsed, be long-lasting, and occur frequently. ${ }^{27}$ The frequency with which an event is recalled may be desired (i.e., as in ruminating to make sense of a situation for approach coping), or undesired (i.e., as in suppression for avoidant coping).

Approach or avoidance-based coping with uncomfortable memory content may result in deferral of insulin initiation. Furthermore, if a stressful memory is undesired and/ or intrusive, then the individual may cope with the memory by avoiding stimuli that conjures it up. For example, if a memory of family member using insulin was stressful, the patient may not want to see a syringe because it acts as stimuli to conjure up the uncomfortable memory. Decisionmaking to initiate insulin may be deferred or if initiated may result in insulin omission in an attempt to avoid or suppress negative images. The result of this decisional conflict over insulin use results in function impairment in diabetes self-management.

Psychological insulin resistance pertains to an individual's fear or negative attitudes toward insulin use that results in an unwillingness or reluctance to take insulin. ${ }^{28}$ Assessing potential barriers for medication adherence is an important element of prescribing practice and routine medication reconciliation. Avoiding the use of insulin as part of one's own self-management plan can result in life-threatening diabetes-related complications.

Limitations of this study include the small sample size making it difficult to detect effects, especially between subject differences on survey scores. The self-report and retrospective method, especially for self-care behavior, lends itself to recall bias, impression management, and social desirability confounding effects. Participants were asked to think about 7 days prior to hospitalization that they felt well enough to follow their self-care regimen. Several factors may have influenced results specific to the hospital context, including interruptions by staff, fatigue, and competing demands of dealing with an acute illness. Finally, because the sample represents those experiencing an acute illness, possibly even diabetes-related, it is possible the memories of a family member's experiences were more prominent than usual. In addition, heightened emotional reactions due to being hospitalized may offer an opportunity for rumination through feelings of guilt, self-blame, or anxiety.

This study does not theoretically address social learning from a general perspective, but is driven by the tenets of the Multigenerational Legacy of Diabetes Theory ${ }^{2,3}$ derived from Rolland's Family Systems Illness Model..$^{14}$ According to Scollan-Koliopoulos et al, ${ }^{3}$ those who consider themselves to be a blood relative develop a sense of genetic susceptibility. In-laws and adopted family members may have a tendency to rationalize that they are not at risk from shared diet or environmental factors because they do not share the same hereditary predisposition. Future studies should compare those who have family-based memory activation and rehearsal of self-care behaviors, with and without a sense of hereditary predisposition based on blood relative status.

\section{Clinical Implications}

From a clinical perspective, one reason it is important to examine individuals' memories of previous generations is because these memories may relate to a different era of diabetes treatment than that experienced by patients today. For example, many individuals recall family member's having poor outcomes after starting insulin, not realizing that the treatment was initiated after complications started as opposed to early enough to prevent them. This results in faulty thinking that the insulin caused the complication.

Probing about the frequency, vividness, and ability to stop the memories of a family member's diabetes-related experiences may offer an opportunity to identify those who are experiencing comorbid depression and anxiety. Once an individual discloses emotional reactions, a dialogue or formal depression and anxiety screening can be employed, since depression itself is an independent predictor of medication adherence. ${ }^{29}$ Clinicians are encouraged to identify and treat individuals for depression and anxiety as is widely advocated for those with diabetes. ${ }^{30,31}$ Providers have an opportunity to identify a patient's strengths and positive coping skills to empower regimen adherence.

In this study, the emotional distress was associated with nonadherence to insulin regimen indicating impaired functionality with medication adherence by way of avoidance, indicating the possibility of a mental disorder. The clinician's role would include further evaluation for anxiety disorder, such as a phobia, adjustment disorder, and/or posttraumatic stress disorder.

Assessing the patient's recollections of a family member's psychological insulin resistance and the individuals own potential for psychological insulin resistance will provide an opportunity to intervene with psychoeducational or therapeutic interventions before insulin is absolutely needed.

Some questions that can be used to screen for the impact of a multigenerational legacy of diabetes on potential insulin adherence include; "How often do you remember your family member being fearful of insulin or actually visualize the family member self-injecting insulin?-How frequent, vivid, or stoppable are the memories" or "How often do you feel sad, mad, angry, depressed, fearful, or guilty about having diabetes?-How about the family member you remember?." The negative implications should be transposed against concrete examples whereby a patient has demonstrated success in self-management behavior to be used as an example of resiliency. 


\section{Conclusion}

The purpose of this study was to estimate the degree to which individuals adhere to insulin regimens in the context of a multigenerational legacy of diabetes. This was done essentially by measuring the nature and quality of vivid, frequent, and uncontrollable memory rehearsal on the development of emotional reactions to having diabetes and those who ruminate over and/or rehearse the memories of a family member's fear of insulin injection are also more likely to experience negative reactions themselves and are subsequently less likely to adhere to their insulin regimen. However, those who ruminate and rehearse, yet do not themselves experience negative reactions, are more likely to adhere to their regimens. The data presented from this study indicates that the negative reactions to having diabetes play a role in shaping the way the next generation reacts to having diabetes and copes with self-care behavior.

Individuals who have a multigenerational legacy of diabetes have an awareness of the family member's experiences with diabetes. For some individual's memories consist of positive content, resulting in positive role-modeling or behavioral responses to control one's own diabetes through self-management. There is no doubt a resiliency and strength for some. In fact, families can and should be relied upon to be actively engaged in family member's self-care effort if mutually desired. ${ }^{32}$ For some, however, the memories may consist of negative content resulting in emotional distress or arousal resulting in avoidance of certain self-care behaviors. For individuals with emotional distress or impaired functioning, time-limited interventions may be useful for promoting insulin adherence. Future studies must address the resilience and strength of a multigenerational legacy of diabetes. For now, clinicians should continue to help patients to focus on how to find examples of resiliency and strength in coping with diabetes self-management to overcome any negative perceptions or recollections of a family member's experiences in the context of a multigenerational legacy of diabetes. For now, clinicians should continue to help patients focus on resiliency and strength in coping with diabetes self-management as a means of overcoming any negative perceptions or recollections in the context of a multigenerational legacy of diabetes. Essentially, upwards of a quarter of patients with diabetes trend toward suboptimal adherence due to negative beliefs about insulin. ${ }^{33}$

\section{Note}

M.S.-K. is the sole author, a family nurse practitioner, and certified diabetes educator at Transformative Health and Behavior Solutions. At the time of this study, the author was Assistant Professor of Medicine at the New Jersey Medical School, Rutgers University formerly, UMDNJ.

\section{Funding}

This project was funded by the Foundation of the University of Medicine and Dentistry of New Jersey.

\section{Conflict of Interest}

None declared.

\section{Acknowledgments}

The author thanks Hackensack University Medical Center and Morristown Memorial Hospital of the Atlantic Health System for instrumentally supporting this study and providing recommendations to strengthen the study. The author would also like to extend gratitude to Kenneth J. Rapp III, MD and Michelle Casey, RN for their assistance with data collection.

\section{References}

1 Centers for Disease Control. (2017). National Diabetes Statistics Report. Available at: http://www.diabetes.org/assets/pdfs/basics/ cdc-statistics-report-2017.pdf. Accessed February 1, 2019

2 Scollan-Koliopoulos MM, O'Connoll KA, Walker E. Multigenerational legacies of diabetes and illness representation: a useful framework for facilitating health behavior. Clin Nurse Spec 2005;19:302-307

3 Scollan-Koliopoulos M, O'Connell KA, Walker EA. Legacy of diabetes and self-care behavior. Res Nurs Health 2007; 30(5):508-517

4 Davies MJ, Gagliardino JJ, Gray LJ, Khunti K, Mohan V, Hughes R. Real-world factors affecting adherence to insulin therapy in patients with type 1 or type 2 diabetes mellitus: a systematic review. Diabet Med 2013;30(5):512-524

5 Sokol MC, McGuigan KA, Verbrugge RR, Epstein RS. Impact of medication adherence on hospitalization risk and healthcare cost. Med Care 2005;43(6):521-530

6 Capoccia K, Odegard PS, Letassy N. Medication adherence with diabetes medication: a systematic review of the literature. Diabetes Educ 2016;42(1):34-71

7 Stratton IM, Adler AI, Neil HA, et al. Association of glycaemia with macrovascular and microvascular complications of type 2 diabetes (UKPDS 35): prospective observational study. BMJ 2000;321(7258) :405-412

8 Gatwood J, Erickson SR. Medication adherence: the search for interventions that work.AmJManag Care2010;16(12):925-926

9 Peyrot M, Barnett AH, Meneghini LF, Schumm-Draeger PM. Insulin adherence behaviours and barriers in the multinational global attitudes of patients and physicians in insulin therapy study. Diabet Med 2012;29(5):682-689

10 Conway MA, Anderson SJ, Larsen SF, et al. The formation of flashbulb memories. Mem Cognit 1994;22(3):326-343

11 Scollan-Koliopoulos M, Walker EA, Bleich D. Perceived risk of amputation, emotions, and foot self-care among adults with type 2 diabetes. Diabetes Educ 2010;36(3):473-482

12 Delgado MR, Locke HM, Stenger VA, Fiez JA. Dorsal striatum responses to reward and punishment: effects of valence and magnitude manipulations. Cogn Affect Behav Neurosci 2003;3(1):27-38

13 Tricomi E, Fiez JA. Information content and reward processing in the human striatum during performance of a declarative memory task. Cogn Affect Behav Neurosci 2012;12(2):361-372

14 Rolland JS, Families, Illness, \& Disability: An Integrative Treatment Model New York: Basic Books

15 Stevens J. Applied Multivariate Statistics for the Social Sciences 4th edition. Mahwah, NJ: Lawrence Erlbaum Associates; 2002

16 Toobert DJ, Hampson SE, Glasgow RE. The summary of diabetes self-care activities measure: results from 7 studies and a revised scale. Diabetes Care 2000;23(7):943-950

17 Broadbent E, Petrie KJ, Main J, Weinman J. The brief illness perception questionnaire. J Psychosom Res 2006;60(6):631-637

18 Scollan-Koliopoulos M, O'Connell KA, Walker EA. The context of a legacy of diabetes: correlates of self-care behavior. Diabetes Educ 2007;33(2):315-324 
19 SPSS Inc. Released 2009. PASW Statistics for Windows, Version 18.0. Chicago: SPSS Inc

20 Myers J, Well A, Research Design and Statistical Analysis 2nd edition. Mahwah: Lawrence Erlbaum Associates; 2003

21 Kihlstrom J, Eich E, Sandbrand D, Tobias B. Emotion and memory: Implications for self-report. In: Stone A, Turkkan J, Bachrach C, Jobe J, Kurtzman H, Cain V, eds. The Science of Self-Report: Implications for Research and Practice. Mahwah, NJ: Lawrence Erlbaum Associates; 2000:81-100

22 Menon G, Yorkston E, The use of memory and contextual cues in the formation of behavioral frequency judgements. In: Stone A, Turkkan J, Bachrach J, Jobe J, Kurtzman H, Cain V, eds. The Science of Self-Report: Implications for Research and Practice. Mahwah, NJ: Lawrence Erlbaum Associates; 2000:63-80

23 Kleinsmith LJ, Kaplan S. Paired-associate learning as a function of arousal and interpolated interval. J Exp Psychol 1963;65:190-193

24 Bradburn N, (; 2000 ). Temporal representation and event dating. In: Stone A, Turkkan J, Bachrach C, Jobe J, Kurtzman H, Cain $\mathrm{V}$, eds. The Science of Self-Report: Implications for Research and Practice Mahwah, NJ: Lawrence Erlbaum Associates; 2000:49-62

25 Lepore SJ. Expressive writing moderates the relation between intrusive thoughts and depressive symptoms. J Pers Soc Psychol 1997;73(5):1030-1037
26 Harris CR, Pashler H. Enhanced memory for negatively emotionally charged pictures without selective rumination. Emotion 2005;5(2):191-199

27 Talarico JM, Rubin DC. Confidence, not consistency, characterizes flashbulb memories. Psychol Sci 2003;14(5):455-461

28 Larkin ME, Capasso VA, Chen CL, et al. Measuring psychological insulin resistance: barriers to insulin use. Diabetes Educ 2008;34(3):511-517

29 Fisher L, Glasgow RE, Strycker LA. The relationship between diabetes distress and clinical depression with glycemic control among patients with type 2 diabetes. Diabetes Care 2010;33(5):1034-1036

30 Jones LE, Doebbeling CC. Depression screening disparities among veterans with diabetes compared with the general veteran population. Diabetes Care 2007;30(9):2216-2221

31 Katon WJ, Von Korff M, Lin EH, et al. The pathways study: a randomized trial of collaborative care in patients with diabetes and depression. Arch Gen Psychiatry 2004;61(10):1042-1049

32 Ahmed Z, Yeasmeen F. Active family participation in diabetes self-care: a commentary. Diabetes Management 2016;6: 104-107

33 Yavuz DG, Ozcan S, Deyneli O. Adherence to insulin treatment in insulin-naïve type 2 diabetic patients initiated on different insulin regimens. Patient Prefer Adherence 2015;9:1225-1231 